\title{
Analyzing the effect of large rotations on the seismic response of structures subjected to foundation local uplift
}

\author{
N. El Abbas ${ }^{1}$, A. Khamlichi ${ }^{2}$, and M. Bezzazi ${ }^{1}$ \\ ${ }^{1}$ Faculty of Science and Techniques Tangier, Department of Physics, Box 416, 90000 Tangier, Morocco \\ ${ }^{2}$ ENSA Tetouan, Department TITM, Box 2222, 93030 Tetouan, Morocco
}

\begin{abstract}
This work deals with seismic analysis of structures by taking into account soil-structure interaction where the structure is modeled by an equivalent flexible beam mounted on a rigid foundation that is supported by a Winkler like soil. The foundation is assumed to undergo local uplift and the rotations are considered to be large. The coupling of the system is represented by a series of springs and damping elements that are distributed over the entire width of the foundation. The non-linear equations of motion of the system were derived by taking into account the equilibrium of the coupled foundation-structure system where the structure was idealized as a single-degree-of-freedom. The seismic response of the structure was calculated under the occurrence of foundation uplift for both large and small rotations. The non-linear differential system of equations was integrated by using the Matlab command ode15s. The maximum response has been determined as function of the intensity of the earthquake, the slenderness of the structure and the damping ratio. It was found that considering local uplift with small rotations of foundation under seismic loading leads to unfavorable structural response in comparison with the case of large rotations.
\end{abstract}

\section{Introduction}

The effect of the foundation uplift on the dynamic response of structures has been investigated by many researchers. Housner [1] was the first to study the problem of structures with uplift in detail and to observe some favorable effect of uplift on structural response magnitude. Meek [2] studied the effects of tipping-uplift on the response of a single-degree-of-freedom (SDOF) system and reported that allowing the SDOF system to tip/uplift altered its natural frequency and led to significant reductions in base reactions and in transverse deformations. Further Meek [3] performed analysis of a core stiffened buildings Meek and concluded that in comparison with a fixed-base core-braced structures, tipping greatly reduces the base shear and moment when subjected to seismic excitation.

Considering the flexibility of the structure and the soil to be represented as a Winkler foundation with large rotations leads to considerable difficulties in the governing equations of motion of the coupled soilstructure system. This is why few studies were dedicated to the complete representation of soil-structure interaction by equations of motion under the hypothesis of large rotation of foundation and the occurrence of $P-\Delta$ effect [4]. Instead simplified equations, consisting of only small rotations of the foundation uplift, have been considered [5].
The first objective of the present paper is to perform analysis of the effect on the seismic response of the structure that result from local uplift of foundation by considering both large and small rotations, but within the context of small deformation of the structure. The seismic response will be determined as function of the intensity of the earthquake, the slenderness of the structure and the damping ratio in vertical vibration of the system with its foundation mat bonded to the supporting elements. Then discrepancies that appear on the response when comparing the small base rotation case and the large base rotation case will be assessed.

The considered coupled soil-structure model takes into account the degrees of freedom related to mat lateral displacement, base vertical displacement and base rotation with this last being large. Derivation of the equations is first conducted then integration of the obtained system of ordinary differential equations is performed.

\section{Materials and methods}

\subsection{Modeling of soil-structure interaction}

The structure is assumed as a beam like mat which can be further characterized by its first mode of vibration. The structure is like this represented by a one degree of 
freedom linear system of mass $m$, lateral stiffness $k$ and lateral damping $c$. The mat is supposed to be mounted on a rigid foundation basis that is assumed to react as a rigid rectangular plate of negligible thickness. The foundation mass denoted $m_{0}$ is taken to be uniformly distributed; the total moment of inertia is designated by $I_{0}$.

The soil-structure interaction takes place at the interface separating the rigid footing and the foundation soil. This interaction can be described by distributed springs and damping elements over the entire width of the foundation, figure 1 gives a schematic representation of the coupled system. The horizontal slippage between the mat and supporting elements is assumed to be negligible. The stiffness per unit length $k_{w}$ and damping per unit length coefficient $c_{w}$ of the foundation model are assumed constant and independent of displacement amplitude or excitation frequency. The base excitation is specified by the horizontal and vertical accelerations due seismic excitation. Under the influence of this excitation, the foundation mat may uplift through an angle $\theta$ and undergo a vertical movement $v$ defined at its centre of gravity in the unstressed position.

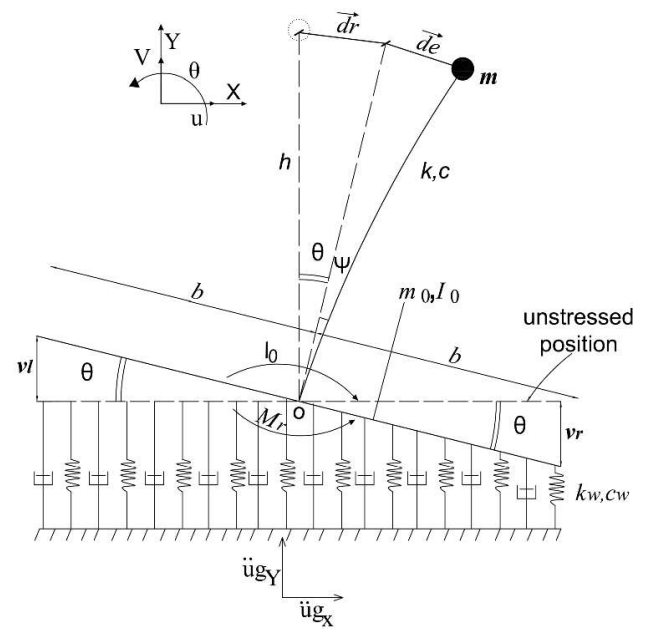

Fig. 1.Flexible structure on Winkler foundation

In figure $1, h$ designates the height of the structure from the base, $M_{r}$ the total moment acting on the base mat, $\vec{d}_{r}$ the rigid horizontal acceleration, $\vec{d}_{e}$ the elastic horizontal displacement of the mat tip relative to the base, $\ddot{\vec{u}}_{g}$ the seismic acceleration, $v$ the vertical displacement of the centre of gravity of the base mat, $\theta$ the angle of rotation of the mat base, $\psi$ the angle rotation of the structure, $b$ half width of foundation mat.

Let us introduce the following notations:

$\omega=\sqrt{k / m}$ natural frequency of the rigidly supported structure,

$\omega_{v}=\sqrt{2 k_{w} b /\left(m+m_{0}\right)}$ vertical vibration frequency of the system with its foundation mat bonded to the supporting elements,

$\alpha=h / b$ slenderness ratio, $\beta=\omega_{v} / \omega$ frequency ratio,

$\gamma=m / m_{0}$ foundation mass to superstructure mass ratio,

$\xi=c /(2 m \omega)$ damping ratio of the rigidly supported structure,

$\xi_{v}=c_{w} b /\left[\left(m+m_{0}\right) \omega_{v}\right]$ damping ratio in vertical vibration of the system with its foundation mat bonded to the supporting elements.

\subsection{Equations of motion}

The equations of motion of the entire system are derived by taking into account the equilibrium of the coupled foundation-mat system. The free body diagram of the system with inertial forces is shown in figure 2 . The three equilibrium equations are:

- Equilibrium of forces acting on each degree of freedom in the horizontal direction: $\sum F_{x}=0$

- Equilibrium of forces in the vertical direction: $\sum F_{y}=0$

- Equilibrium of moments about the center of the foundation of the mat: $\sum M_{z}=0$

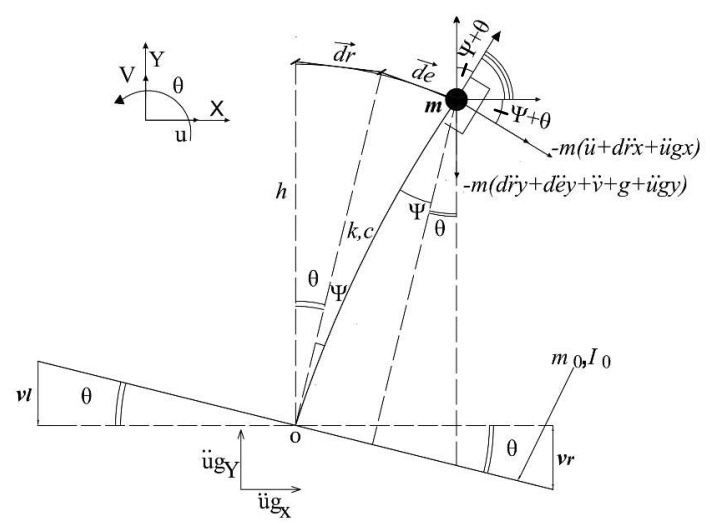

Fig. 2. Free body diagram of the system with uplift showing the considered dependent and independent degrees of freedom

\subsubsection{Equations of motion for large rotations}

Considering the equilibrium of forces in the lateral direction, the equation of motion in terms of the mat tip lateral displacement writes:

$$
m\left(\ddot{d}_{e x}+\ddot{d}_{r x}\right) \cos (\theta+\psi)+c \dot{d}_{e x}+k d_{e x}=-m \ddot{u}_{g x}
$$

with

$$
\begin{aligned}
\ddot{d}_{r x} & =\frac{h \ddot{\theta}}{2}\left[3 \cos \left(\frac{3 \theta}{2}\right)-\cos \left(\frac{\theta}{2}\right)\right] \\
& +\frac{h \dot{\theta}^{2}}{4}\left[-9 \sin \left(\frac{3 \theta}{2}\right)+\sin \left(\frac{\theta}{2}\right)\right]
\end{aligned}
$$

The equilibrium of forces in the vertical direction can be written as 
$\left(m+m_{0}\right)\left(\ddot{v}+\ddot{d}_{e y}+\ddot{d}_{r y}\right)+F_{v}=-\left(m+m_{0}\right) g+\left(m+m_{0}\right) \ddot{u}_{g y}$

with

$$
\begin{aligned}
F_{v}= & \int_{-b}^{b}\left(k_{w} v_{(\ell, r)}+c_{w} \dot{v}_{(\ell, r)}\right) d x \\
\ddot{d}_{r y}= & \frac{h \ddot{\theta}}{2}\left[\sin \left(\frac{\theta}{2}\right)-3 \sin \left(\frac{3 \theta}{2}\right)\right] \\
& +\frac{h \dot{\theta}^{2}}{4}\left[\cos \left(\frac{\theta}{2}\right)-9 \cos \left(\frac{3 \theta}{2}\right)\right] \\
\ddot{d}_{e y}= & -h \cos (\theta)\left(-\psi^{2} \dot{\theta}+\dot{\psi}+2 \dot{\psi}^{2}+2 \psi \ddot{\psi}+\dot{\psi} \dot{\theta}+\psi \ddot{\theta}\right), \\
& +h \sin (\theta)\left(4 \psi \dot{\psi} \dot{\theta}+\psi \dot{\theta}^{2}+\psi^{2} \ddot{\theta}-\ddot{\psi}\right)
\end{aligned}
$$

The vertical displacements at the edges of the foundation mat, see figure3, measured from the initial unstressed positions are:

$$
v_{i}=v \pm x \sin (\theta), i=l, r
$$

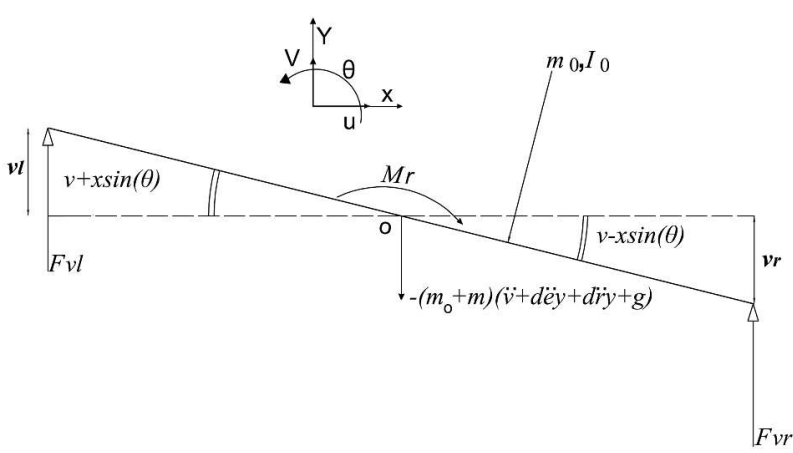

Fig. 3. Free body diagram for the base

In equation (4), $F_{v}$ is the total vertical force acting on the base mat. This force is obtained as

$$
F_{v}=k_{w} \int_{-b}^{b}(v \pm x \sin (\theta)) d x+c_{w} \int_{-b}^{b}(\dot{v} \pm x \dot{\theta} \cos (\theta)) d x
$$

Because the Winkler foundation cannot extend above its initial unstressed position an edge of the foundation mat would uplift at the time instant when [1]:

$v_{i}(t)>0, i=l, r$

Calculating the integral in equation (8) yields the following equation:

$$
\begin{aligned}
& \ddot{v}=-\left(1-\varepsilon_{1}^{2}\right) \varepsilon_{2} \xi_{v} \beta \omega \frac{b}{2}\left[\dot{\theta} \cos (\theta)+\frac{k_{w}}{c_{w}} \sin (\theta)\right] \\
& -\left(1+\varepsilon_{1}\right) \xi_{v} \beta \omega\left(\dot{v}+\frac{k_{w}}{c_{w}} v\right)-\left(\ddot{d}_{r y}+\ddot{d}_{e y}\right)-\mathrm{g}+\ddot{u}_{g y}
\end{aligned}
$$

with $\varepsilon_{1}=\left\{\begin{array}{l}1 \quad \text { contact at both edges } \\ \frac{\varepsilon_{2} v}{b \sin (\theta)} \text { one edge is uplifted }\end{array}\right.$

$\varepsilon_{2}= \begin{cases}-1 & \text { left edge uplifted } \\ 0 & \text { contact at both edges } \\ 1 & \text { right edge uplifted }\end{cases}$

Taking the resultant moment about the centre of the foundation of the base mat, the following equation is readily obtained:

$$
I_{0} \ddot{d}_{r x}+h m\left(\ddot{d}_{r x}+\ddot{d}_{e x}\right) \cos (\theta+\psi)+M_{r}=m h \ddot{u}_{\boldsymbol{g x}}
$$

where $M_{r}$ is the resistant moment which represents the global action of spring and dashpot system acting on the foundation base. It is derived by considering the forces applied on the free body diagram of the base mat as:

$$
\begin{aligned}
& M_{r}=k_{w} \int_{-b}^{b}\left[ \pm v x \pm x^{2} \sin (\theta)\right] d x+ \\
& c_{w} \int_{-b}^{b}\left[ \pm x v \pm x^{2} \dot{\theta} \cos (\theta)\right] d x
\end{aligned}
$$

The integral in equation (14) results in the following equation:

$$
\begin{aligned}
\ddot{\theta}= & \frac{1}{\frac{h}{2}\left[3 \cos \left(\frac{3 \theta}{2}\right)-\cos \left(\frac{\theta}{2}\right)\right]}\left\{-\frac{h \theta^{2}}{4}\left[-9 \sin \left(\frac{3 \theta}{2}\right)+\sin \left(\frac{\theta}{2}\right)\right]\right. \\
& +\frac{3 h}{\gamma b^{2}}\left(2 \xi \omega \dot{u}+\omega^{2} u\right)-\left(1-\varepsilon_{1}^{2}\right) \frac{3 \varepsilon_{2} c_{w}}{2 m_{0}}\left(\dot{v}+\frac{k_{w}}{c_{w}} v\right) \\
& -\left(1+\varepsilon^{3}{ }_{1}\right) \frac{c_{w} b}{m_{0}}\left[\dot{\theta} \cos (\theta)+\frac{k_{w}}{c_{w}} \sin (\theta)\right]
\end{aligned}
$$

The equations of motion of the system in case of large rotation are formed by equations (1), (10) and (15).

\subsubsection{Equations of motion for small rotations}

The equations of motion of the system under hypothesis of a small rotation of the foundation are obtained by using the same approach used in the case of large rotation and by letting the following approximations:

$$
\begin{aligned}
& \cos (\psi+\theta) \approx 1 \\
& \sin (\psi+\theta) \approx \psi+\theta
\end{aligned}
$$

The three final equations of motion are then:

$$
m\left(d_{e x}+h \ddot{\theta}\right)+c \dot{d}_{e x}+k d_{e x}=-m \ddot{u}_{g x}
$$




$$
\begin{aligned}
& \ddot{v}=-\left(1-\varepsilon_{1}^{2}\right) \varepsilon_{2} \xi_{v} \beta \omega \frac{b}{2}\left(\dot{\theta}+\frac{k_{w}}{c_{w}} \theta\right)- \\
& \left(1+\varepsilon_{1}\right) \xi_{v} \beta \omega\left(\dot{v}+\frac{k_{w}}{c_{w}} v\right)-\left(\ddot{d}_{r x}+\ddot{d}_{e x}\right)-\mathrm{g}+\ddot{u}_{g y} \\
& \ddot{\theta}=-\dot{\theta}^{2}\left(-9 \frac{3 \theta}{2}+\frac{\theta}{2}\right)+\frac{12}{\gamma b^{2}}\left(2 \xi \omega \dot{u}+\omega^{2} u\right) \\
& -\left(1-\varepsilon_{1}^{2}\right) \frac{6 \varepsilon_{2} c_{w}}{h m_{0}}\left(\dot{v}+\frac{k_{w}}{c_{w}} v\right)-\left(1+\varepsilon^{3}{ }_{1}\right) \frac{4 c_{w} b}{h m_{0}}\left(\dot{\theta}+\frac{k_{w}}{c_{w}}\right)
\end{aligned}
$$

with

$\varepsilon_{1}= \begin{cases}1 & \text { contact at both edges } \\ \frac{\varepsilon_{2} v}{b \theta} & \text { one edge is uplifted }\end{cases}$

and $\varepsilon_{2}$ having the same definition as in equation (12).

Finally, the equations of motion of the system in the both cases are:

- For large rotation they are formed by equations (1), (10) and (15).

- For small rotation they are formed by equations (18), (19) and (20).

These systems of ordinary differential equations are highly nonlinear. Their numerical integration can be achieved iteratively as the form of this system is not a priori known because of the conditions corresponding to equations (11), (12) et (21).

Integrating the three-non-linear ordinary system of differential equations by using the Matlab command ode $15 \mathrm{~s}$ enables to calculate the response of the structure and to perform parametric studies.

\section{Results}

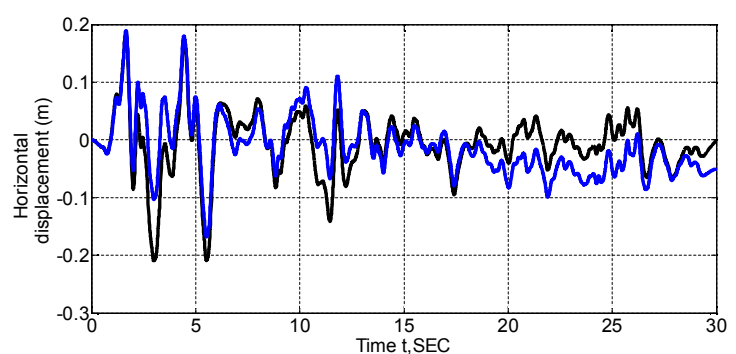

(a)

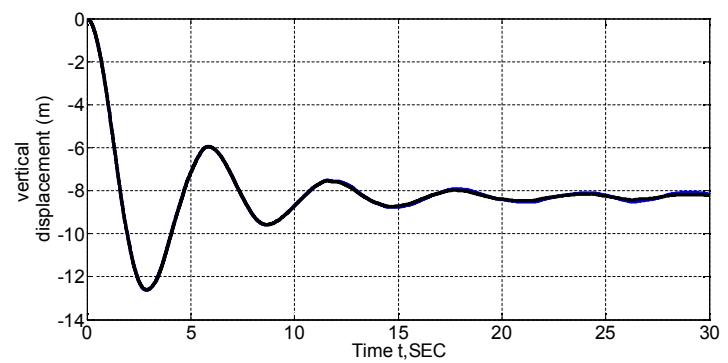

(b)

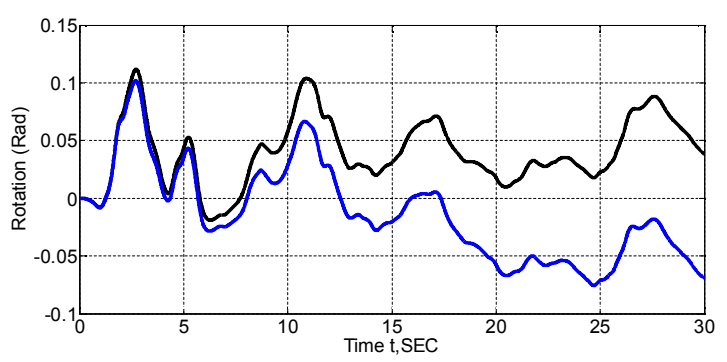

(c)

Fig. 4. Response of the structure under El Centro ground motion: (a) horizontal displacement; (b) vertical displacement; (c) base rotation; blue color is for large rotation of the base and black corresponds to the small rotation of the base

The seismic responses of the considered system are shown in figure 4 for the two hypothesis small and large rotation of foundation. The results present in terms of the lateral displacement of the structure, the foundation rotation and vertical movement to its center of gravity.

\section{Conclusions}

The effect of base uplift on the maximum response of a flexible structure which was taken to set up on a Winkler like foundation has been determined as function of the slenderness of the structure and the damping ratio in vertical vibration of the system with its foundation mat bonded to the supporting elements.

The obtained results lead to some discrepancies between the two cases: large and small rotations. Since the numerical cost is almost the same for the two hypotheses, the general case of large rotations can be considered in order for instance to integrate the $P-\Delta$ effect.

\section{References}

1. G. W. Housner, J. Bulletin of the Seismological Society of America, 53, 403-417 (1963)

2. J.W. Meek, J. Struct. Div., 101, 1297-1311 (1975)

3. J. W. Meek , J. Earthquake Engineering \& Structural Dynamics, 6, 437-454 (1978)

4. G. Oliveto, I. Cali, A. Greco, J. Earthquake Engineering \& Structural Dynamics, 32, 369-393 (2003)

5. M. Apostolou, N. Gerolymos, J. Bulletin of Earthquake Engineering, 8, 309-326 (2009) 